\title{
CHARACTER TABLE GROUPS AND EXTRACTED SIMPLE AND CYCLIC POLYGROUPS
}

\author{
Sara Sekhavatizadeh ${ }^{1}$, Mohamad Mehdi Zahedi ${ }^{2}$, Ali \\ IRANMANESH ${ }^{3}$ \\ ${ }^{1}$ Department of Mathematics, Tarbiat Modares University, Tehran, Iran, \\ s.sekhavatizadeh@modares.ac.ir \\ ${ }^{2}$ Graduate University of Advanced Technology, Kerman, Iran, \\ zahedi_mm@kgut.ac.ir \\ ${ }^{3}$ Department of Mathematics, Tarbiat Modares University, Tehran, Iran, \\ iranmanesh@modares.ac.ir
}

\begin{abstract}
Let $G$ be a finite group and $\hat{G}$ be the set of all irreducible complex characters of $G$. In this paper, we consider $\hat{G}$ as a polygroup. We call that $\hat{G}$ simple if it has no proper normal subpolygroup and show that if $\hat{G}$ is a single power cyclic polygroup, then $\hat{G}$ is a simple polygroup and hence the polygroups wich are indeuced by symmetric group and alternating group are simple. Also, we prove that if $G$ is a non-abelian simple group, then $\hat{G}$ is a single power cyclic polygroup. Moreover, we classify $\hat{D}_{2 n}$ for all $n$. Also, we investigate the same property for two other groups. Key words and Phrases: Character of group, hypergroup, polygroup, cyclic hypergroup, fundamental relation.
\end{abstract}

\section{INTRODUCTION}

Let $G$ be a finite group and $\operatorname{Irr}(\mathrm{G})=\left\{\chi_{1}, \chi_{2}, \ldots, \chi_{\mathrm{k}}\right\}$ be the set of all irreducible characters of $G$. Brauer [1] introduced the idea of studying character tables considering them as square arrays of complex numbers satisfying certain conditions. Afterwards, Comer [2] described several hypergroup constructions based on assumptions which arise in the study of symmetry. In particular, he showed that a natural hypergroup is associated with every character algebra. Hence the hypergroup suggests itself as a tool for group theorists. These concepts provide a new language in which groups and their character tables can be fruitfully discussed.

Roth in [11] studied canonical hypergroups $\left\langle\hat{G}, *, \chi_{1},{ }^{-}>\right.$, where $\hat{G}=\operatorname{Irr}(\mathrm{G})$, $\chi_{1}$ is the trivial character and the product $\chi_{i} * \chi_{j}$ is the set of those irreducible constituents which appear in the element wise product $\chi_{i} \chi_{j}$. Furthermore, $\bar{\chi}$, the

2020 Mathematics Subject Classification: 1257

Received: 16-02-2018, accepted: 26-10-2018. 
complex conjugate of $\chi$, is the inverse of $\chi$. Canonical hypergroups were introduced by Mittas [10] and later, Corsini in [3] analyses a particular type of canonical hypergroups and their homomorphisms. Afterwards, Comer introduced this class of hypergroups independently, using the name of polygroups and pointed out that polygroups have application in color schemes and combinatorics[2]. We call the polygroup $\hat{G}$ the "character polygroup". The structure of polygroups is similar to groups, since identity and inverse elements exist in polygroups. So, we say $\mathcal{P}=\left\langle P, \cdot, e,^{-1}>\right.$, is a polygroup if $P$ is a non-empty set, $e \in P,{ }^{-1}$ is a unitary operation on $P, \cdot$ maps $P \times P$ into the set of all non-empty subsets of $P$, and the following axioms hold for all $x, y, z \in P$ :

(1) $(x \cdot y) \cdot z=x \cdot(y \cdot z)$;

(2) $e \cdot x=x \cdot e=x$;

(3) $x \in y \cdot z$ implies that $y \in x \cdot z^{-1}$ and $z \in y^{-1} \cdot x$.

A non-empty subset $K$ of a polygroup $P$ is a subpolygroup of $P$ if

(1) $a, b \in K$ implies that $a \cdot b \subseteq K$,

(2) $a \in K$ implies that $a^{-1} \in K$.

Since polygroups have properties close to groups, concepts such as normal subgroups and isomorphism theorems are defined for them . A subpolygroup $N$ of a polygroup $P$ is normal in $P$ if $a^{-1} N a \subseteq N$, for all $a \in P$ see [4]. We say that the polygroup $P$ is simple if it does not have any proper normal subpolygroups. So if $G$ is a simple group, then $\hat{G}$ is a simple polygroup. It is clear that $\hat{G}$ is always a normal subpolygroup and the trivial subpolygroup $\left\{\chi_{1}\right\}$ is not a normal subpolygroup of $\hat{G}$. The Classification of simple groups has been of interest to mathematicians for many years.

Roth in [11], showed that the mapping $K \longrightarrow \frac{\widehat{G}}{K}$ yields a one to one correspondence between the set of all normal subgroups of $G$ and the set of subpolygroups of $\hat{G}$. In this paper, we investigate the simplicity of a character polygroup $\hat{G}$. In fact, we show that if $\hat{G}$ is a single power cyclic polygroup, then it is a simple polygroup. The proof of this result is carried out using the fundamental relations on hypergroups. Cyclic hypergroups are a certain subclass of hypergroups. Cyclic hypergroups were first initiated by Wall [15] and afterwards have been studied by Vougiouklis [13], Konguetsof et al. [6] and Leoreanu [8]. The hypergroup $(H, \circ)$ is called cyclic with finite period respect to $h \in H$ if there exists a positive integer $s \in \mathbb{Z}^{+}$, such that

$$
H=h^{1} \cup h^{2} \cup \ldots \cup h^{s},
$$

where $h^{t}=\underbrace{h \circ h \circ \cdots \circ h}_{t \text { times }}$. The minimum of all such positive integers $s$ is called the period of the generator $h$. If there exists $k \in \mathbb{Z}^{+}$, such that

$$
H=h^{k},
$$

then $H$ is called a single power cyclic hypergroup and $h$ is a generator of $H$. The minimum of all such positive integer $k$ is called the period of the generator $h$. In [12], we showed that $\hat{S}_{n}$ for $n \geq 3$ and $\hat{A_{n}}$ for $n \geq 4$ are single power cyclic 
polygroups. An obvious conclusion of this result is that $\hat{S}_{n}$ for $n \geq 3$ and $\hat{A}_{n}$ for $n \geq 4$ are simple polygroups. Also, we classify all subpolygroups of $\hat{D}_{2 n}$ and we show that $\hat{D}_{2 n}$ for even $n$, has exactly one normal subpolygroup and for odd $n$, is simple. This is a counter example that shows that the converse of our claim is not true in general. Also, we show that the polygroups $\hat{T}_{4 n}$ and $\hat{U}_{6 n}$ are cyclic with finite period. Moreover, we prove that if $G$ is a non-abelian simple group, then $\hat{G}$ is a single power cyclic polygroup. Throughout this paper, $\chi_{1}$ is the trivial character and for an irreducible character $\chi_{i}$, we denote $\underbrace{\chi_{i} * \chi_{i} * \ldots * \chi_{i}}_{t \text { times }}$ by $\chi_{i}^{t}$, where the hyperoperation $*$ is as above.

\section{PRELIMINARIES}

In this section, we recall some definitions and facts about hypergroups and characters of finite groups, referring to $[5,4]$ and [11].

Let $\operatorname{Irr}(G)=\left\{\chi_{1}, \chi_{2}, \ldots, \chi_{k}\right\}$, where $\chi_{i}$ for $1 \leq i \leq k$ are all complex irreducible characters of $G$. Then for any two characters $\chi_{i}, \chi_{j}$ of $G,\left(\chi_{i}, \chi_{j}\right)$ denotes the usual inner product:

$$
\left(\chi_{i}, \chi_{j}\right)=\frac{1}{|G|} \sum_{g \in G} \chi_{i}(g) \chi_{j}\left(g^{-1}\right)
$$

Theorem 2.1 ([5], Theorem 16.4). (Orthogonality relations)

Let $\left\{\chi_{1}, \chi_{2}, \ldots, \chi_{k}\right\}$ be the set of all irreducible characters of $G$, and let $g_{1}, \ldots, g_{k}$ be representatives of the conjugacy classes of $G$. Then the following relations hold for any $r, s \in\{1, \ldots, k\}$ :

(1) The row orthogonality relations:

$$
\sum_{i=1}^{k} \frac{\chi_{r}\left(g_{i}\right) \overline{\chi_{s}\left(g_{i}\right)}}{\left|C_{G}\left(g_{i}\right)\right|}=\delta_{r s}
$$

(2) The column orthogonality relations:

$$
\sum_{i=1}^{k} \chi_{i}\left(g_{r}\right) \overline{\chi_{i}\left(g_{s}\right)}=\delta_{r s}\left|C_{G}\left(g_{r}\right)\right| .
$$

Theorem 2.2 ([5], Theorem 19.10). Let $\chi$ be a faithful character of $G$ and suppose $\chi(g)$ takes on exactly $m$ different values for $g \in G$. Then every $\psi \in \operatorname{Irr}(G)$ is a constituent of one of the characters $(\chi)^{j}$ for $0 \leq j<m$. 
Theorem 2.3 ([5], Theorem 19.18). Let $\chi_{1}, \ldots, \chi_{a}$ be the distinct irreducible characters of $G$ and let $\psi_{1}, \ldots, \psi_{b}$ be the distinct irreducible characters of $H$. Then $G \times H$ has precisely ab distinct irreducible characters, and these are

$$
\chi_{i} \times \psi_{j}(1 \leq i \leq a, 1 \leq j \leq b)
$$

and

$$
\chi_{i} \times \psi_{j}(g, h)=\chi_{i}(g) \psi_{j}(h)(g \in G, h \in H) .
$$

Theorem 2.4 ([5], 18.3). The character table of $G=D_{2 n}=<a, b: a^{n}=1, b^{2}=$ $1, b^{-1} a b=a^{-1}>$ is as follows (where $\epsilon=e^{\frac{2 \pi i}{n}}$ ):

TABLE 1. Character table of $D_{2 n}$ for $n=2 m$ (where $\left.1 \leq j \leq m-1\right)$

\begin{tabular}{c|ccccc} 
Class & 1 & $a^{m}$ & $a^{r}(1 \leq r \leq m-1)$ & $b$ & $a b$ \\
\hline$\left|C_{G}\left(g_{i}\right)\right|$ & $2 n$ & $2 n$ & $n$ & 4 & 4 \\
\hline$\chi_{1}$ & 1 & 1 & 1 & 1 & 1 \\
$\chi_{2}$ & 1 & 1 & 1 & -1 & -1 \\
$\chi_{3}$ & 1 & $(-1)^{m}$ & $(-1)^{r}$ & 1 & -1 \\
$\chi_{4}$ & 1 & $(-1)^{m}$ & $(-1)^{r}$ & -1 & 1 \\
$\psi_{j}$ & 2 & $2(-1)^{j}$ & $\epsilon^{j r}+\epsilon^{-j r}$ & 0 & 0
\end{tabular}

TABLE 2. Character table of $D_{2 n}$ for odd integer $n$ (where $1 \leq j \leq \frac{n-1}{2}$ )

\begin{tabular}{c|ccc} 
Class & 1 & $a^{r}\left(1 \leq r \leq \frac{n-1}{2}\right)$ & $b$ \\
\hline$\left|C_{G}\left(g_{i}\right)\right|$ & $2 n$ & $n$ & 2 \\
\hline$\chi_{1}$ & 1 & 1 & 1 \\
$\chi_{2}$ & 1 & 1 & -1 \\
$\psi_{j}$ & 2 & $\epsilon^{j r}+\epsilon^{-j r}$ & 0
\end{tabular}

Theorem 2.5 ([5], Exercise 18.3). The character table of $G=T_{4 n}=<a, b: a^{2 n}=$ $1, a^{n}=b^{2}, b^{-1} a b=a^{-1}>$ is as follows (where $1 \leq j \leq n-1, \epsilon=e^{\frac{2 \pi i}{2 n}}$ ):

TABLE 3. Table of non-linear characters of $T_{4 n}$

\begin{tabular}{c|ccccc} 
Class & 1 & $a^{n}$ & $a^{r}(1 \leq r \leq n-1)$ & $b$ & $a b$ \\
\hline$\left|C_{G}\left(g_{i}\right)\right|$ & $4 n$ & $4 n$ & $2 n$ & 4 & 4 \\
\hline$\psi_{j}$ & 2 & $2(-1)^{j}$ & $\epsilon^{j r}+\epsilon^{-j r}$ & 0 & 0
\end{tabular}


TABLE 4. Table of linear characters of $T_{4 n}$ for even integer $n$

\begin{tabular}{c|ccccc} 
Class & 1 & $a^{n}$ & $a^{r}(1 \leq r \leq n-1)$ & $b$ & $a b$ \\
\hline$\left|C_{G}\left(g_{i}\right)\right|$ & $4 n$ & $4 n$ & $2 n$ & 4 & 4 \\
\hline$\chi_{1}$ & 1 & 1 & 1 & 1 & 1 \\
$\chi_{2}$ & 1 & 1 & 1 & -1 & -1 \\
$\chi_{3}$ & 1 & 1 & $(-1)^{r}$ & 1 & -1 \\
$\chi_{4}$ & 1 & 1 & $(-1)^{r}$ & -1 & 1
\end{tabular}

TABLE 5. table of linear characters of $T_{4 n}$ for odd integer $n$

\begin{tabular}{c|ccccc} 
Class & 1 & $a^{n}$ & $a^{r}(1 \leq r \leq n-1)$ & $b$ & $a b$ \\
\hline$\left|C_{G}\left(g_{i}\right)\right|$ & $4 n$ & $4 n$ & $2 n$ & 4 & 4 \\
\hline$\chi_{1}$ & 1 & 1 & 1 & 1 & 1 \\
$\chi_{2}$ & 1 & 1 & 1 & -1 & -1 \\
$\chi_{3}$ & 1 & -1 & $(-1)^{r}$ & $i$ & $-i$ \\
$\chi_{4}$ & 1 & -1 & $(-1)^{r}$ & $-i$ & $i$
\end{tabular}

TABLE 6. Character table of $U_{6 n}$

\begin{tabular}{c|ccc} 
Class & $a^{2 r}$ & $a^{2 r} b$ & $a^{2 r+1}$ \\
\hline$\left|C_{G}\left(g_{i}\right)\right|$ & $6 n$ & $3 n$ & $2 n$ \\
\hline$\chi_{j}$ & $\epsilon^{2 j r}$ & $\epsilon^{2 j r}$ & $\epsilon^{j(2 r+1)}$ \\
$0 \leq j \leq 2 n-1$ & & & \\
$\psi_{k}$ & $2 \epsilon^{2 k r}$ & $-\epsilon^{2 k r}$ & 0 \\
$0 \leq k \leq n-1$ & & &
\end{tabular}

Theorem 2.6 ([5], Exercise 18.4). The character table of $G=U_{6 n}=<a, b: a^{2 n}=$ $b^{3}=1, a^{-1} b a=b^{-1}>$ is as follows (where $0 \leq r \leq n-1, \epsilon=e^{\frac{2 \pi i}{2 n}}$ ):

Theorem 2.7 ([11], Theorem 2.3). Let $M$ be a non-empty subset of $\hat{G}$. Then $M$ is a subpolygroup of $\hat{G}$ if $\chi * \psi \subseteq M$ whenever $\chi, \psi \in M$.

Irreducible characters of $G$ containing $K$ in their kernel are easily identified with the irreducible characters of $\frac{G}{K}$. Thus we regard $\frac{\widehat{G}}{K}$ as a subset of $\hat{G}$ and it is easily seen to be a subpolygroup.

Theorem 2.8 ([11], Theorem 2.2). The mapping $K \longrightarrow \frac{\widehat{G}}{K}$ yields a one-one correspondence between the set of normal subgroups of $G$ and the set of subpolygroups of $\hat{G}$. 
Now we summarize some basic facts a bout equivalence relations. Let $H$ be a hypergroup and $R \subseteq H \times H$ be an equivalence relation on $H$. For non-empty subsets $A$ and $B$ of $H$, we define

$A \bar{R} B \Leftrightarrow \forall a \in A, \exists b \in B$ such that $a R b$ and $\forall b^{\prime} \in B, \exists a^{\prime} \in A$ such that $a^{\prime} R b^{\prime}$;

$$
A \overline{\bar{R}} B \Leftrightarrow a R b, \forall a \in A, \forall b \in B .
$$

The relation $R$ is called:

(1) regular on the left (on the right) if

$x R y \Rightarrow a \circ x \bar{R} a \circ y(x \circ a \bar{R} y \circ a$, respectively), for all $x, y, a \in H$.

(2) strongly regular on the left (on the right) if

$x R y \Rightarrow a \circ x \overline{\bar{R}} a \circ y(x \circ a \overline{\bar{R}} y \circ a$, respectively $)$, for all $x, y, a \in H$.

Moreover, $R$ is called regular (strongly regular) if it is regular (strongly regular) on the right and on the left.

Theorem 2.9 ([4], Theorem 2.5.2). Let $(H, \circ)$ be a hypergroup and $R$ be an equivalence relation on $H$.

(1) If $R$ is regular, then $\frac{H}{R}$ is a hypergroup, with respect to the following operation: $\bar{x} \otimes \bar{y}=\{\bar{z} \mid z \in x \circ y\}$;

(2) If the above operation is well defined on $\frac{H}{R}$, then $R$ is regular.

Theorem 2.10 ([4], Theorem 2.5.5). Let $(H, \circ)$ be a hypergroup and $R$ be an equivalence relation on $H$.

(1) If $R$ is strongly regular, then $\frac{H}{R}$ is a group, with respect to the following operation: $\bar{x} \otimes \bar{y}=\{\bar{z} \mid z \in x \circ y\}$;

(2) If the above operation is well defined on $\frac{H}{R}$, then $R$ is strongly regular.

Let $N$ be a normal subpolygroup of $P$. Then we define the relation $x \equiv$ $y(\bmod N)$ if and only if $x y^{-1} \cap N \neq \phi$. This relation is denoted by $x N_{P} y$.

Lemma 2.11 ([4], Lemma 3.3.6). The relation $N_{P}$ is an equivalence relation.

Let $N_{P}(x)$ be the equivalence class of the element $x \in P$. Suppose that $[P: N]=\left\{N_{P}(x) \mid x \in P\right\}$. On $[P: N]$ we consider the hyperoperation $\odot$ defined as follows:

$$
N_{P}(x) \odot N_{P}(y)=\left\{N_{P}(z) \mid z \in N_{P}(x) N_{P}(y)\right\} .
$$

For a subpolygroup $K$ of $P$ and $x \in P$, denote the right coset of $K$ by $K x$ and let $\frac{P}{K}$ be the set of all right cosets of $K$ in $P$.

Lemma 2.12 ([4], Lemma 3.3.7). If $N$ is a normal subpolygroup of $P$, then $N x=$ $N_{P}(x)$. 
Theorem 2.13 ([4], Corollary 3.3.14). If $N$ is a normal subpolygroup of $P$, then $<\frac{P}{N}, \odot, N,^{-I}>$ is a polygroup, where $N x \odot N y=\{N z \mid z \in x y\}$ and $(N x)^{-I}=$ $N x^{-1}$.

\section{SIMPLE CHARACTER POLYGROUPS}

In this section, we classify all subpolygroups of $\hat{D}_{2 n}$ and we show that when $n$ is an even integer, $\hat{D}_{2 n}$ is not simple and for odd $n$ it is simple. Also, we show that if $\hat{G}$ is a single power cyclic polygroup, then $\hat{G}$ is simple and in consequence $\hat{S}_{n}$ for $n \geq 3$ and $\hat{A_{n}}$ for $n \geq 4$ are simple.

Theorem 3.1. The polygroup $\hat{D}_{2 n}$ for even integer $n=2 m$ has $t+3$ subpolygroups, where $t$ is the number of divisors of $n$.

Proof. By Theorem 2.8, the set of subpolygroups of $\hat{D}_{2 n}$ is in one to one correspondence with the set of normal subgroups of $D_{2 n}$. We know that the proper normal subgroups of $D_{2 n}$ are $\left\langle a^{i}>,<a^{2}, b>\right.$ and $\left\langle a^{2}, a b>\right.$ where $\left.i\right| n$. Now to obtain the subpolygroups $N_{i}$ related to $\left\langle a^{i}\right\rangle$ consider two cases according to the parity of $i$ and using Table 1 :

a) Suppose that $i$ is an even integer. Then

$$
N_{i}=\left\{\chi_{1}, \chi_{2}, \chi_{3}, \chi_{4}, \psi_{k j}\right\} \text { where } i j=n, 1 \leq k j \leq m-1 .
$$

b) Suppose that $i$ is an odd integer. Then

$$
N_{i}=\left\{\chi_{1}, \chi_{2}, \psi_{k j}\right\} \text { where } i j=n, 1 \leq k j \leq m-1 .
$$

The two remained subpolygroups of $\hat{D}_{2 n}$ are $\left\{\chi_{1}, \chi_{3}\right\},\left\{\chi_{1}, \chi_{4}\right\}$. Therefore, all proper subpolygroups of $\hat{D}_{2 n}$ are obtained.

Theorem 3.2. The polygroup $\hat{D}_{2 n}$ for an even integer $n$ has exactly one normal subpolygroup.

Proof. We claim that $N_{m}$ is the only normal subpolygroup of $\hat{D}_{2 n}$. Let $N_{i} \neq N_{m}$ be a subpolygroup of $\hat{D}_{2 n}$. By definition, $N_{i}$ is normal if for every $\psi_{j}, \psi_{j} N_{i} \overline{\psi_{j}} \subseteq$ $N_{i}$. But the complex conjugate of $\psi_{j}$ is equal to $\psi_{j}$ and hence $N_{i}$ is normal if, $\psi_{j}{ }^{2} N_{i} \subseteq N_{i}$. On the other hand, for each $j$ according to Table 1 and using the orthogonality relations we have

$$
\psi_{j}{ }^{2}=\chi_{1}+\chi_{2}+\psi_{k} \text { for some even integer } k .
$$

Also, $\psi_{1}{ }^{2}=\chi_{1}+\chi_{2}+\psi_{2}$. Hence $\psi_{2}$ is a constituent of $\psi_{1}{ }^{2}$. But $\psi_{2}$ just belongs to subpolygroup $N_{m}$, therefore $N_{i}$ is not normal. Now we show that $N_{m}$ is normal. By $(1), \psi_{j}{ }^{2} \subseteq N_{m}$ and hence by Theorem 2.7, $\psi_{j}{ }^{2} N_{m} \subseteq N_{m}$. Therefore, $N_{m}$ is normal.

Theorem 3.3. The polygroup $\hat{D}_{2 n}$ for odd integer $n$ has $t$ proper subpolygroups where $t$ is the number of divisors of $n$. 
Proof. We know that the normal subgroups of $D_{2 n}$ are $<a^{i}>$ where $i \mid n$. Hence by Theorem 2.8 and Table 2, the subpolygroups of $\hat{D}_{2 n}$ are

$$
N_{i}=\left\{\chi_{1}, \chi_{2}, \psi_{k j}\right\} \text { where } i j=n, 1 \leq k j \leq \frac{n-1}{2} .
$$

Definition 3.4. We say that the polygroup $P$ is simple if it does not have any proper normal subpolygroups.

Theorem 3.5. The polygroup $\hat{D}_{2 n}$ for odd integer $n$ is simple.

Proof. It is clear that $\psi_{1}$ is faithful. So for every $i, \psi_{1} \notin N_{i}$. On the other hand, ${\psi_{1}}^{2}=\chi_{1}+\chi_{2}+\psi_{j}$ for some $\psi_{j}$. Now let $\psi_{j}$ be a non-faithful irreducible character. Then $\psi_{1}^{2}\left(a^{r}\right)=1+1+2=4$ for some $r$. Thus $\psi_{1}\left(a^{r}\right)= \pm 2$. It is clear that $\psi_{1}\left(a^{r}\right) \neq 2$. If $\psi_{1}\left(a^{r}\right)=-2$, then $a^{r} \in Z\left(\psi_{1}\right)=Z\left(D_{2 n}\right)=\{1\}$ and it is a contradiction. Thus $\psi_{j}$ is a faithful irreducible character. So $\psi_{1}{ }^{2}$ does not lie in any subpolygroup. Therefore, the polygroup $\hat{D}_{2 n}$ does not have any normal subpolygroups.

Definition 3.6. For all $n \geq 1$, we define the relation $\beta_{n}$ on a semihypergroup $H$, as follows:

$$
a \beta_{n} b \Leftrightarrow \exists\left(x_{1}, \ldots, x_{n}\right) \in H^{n}:\{a, b\} \subseteq \prod_{i=1}^{n} x_{i}, \text { and } \beta=\bigcup_{n \geq 1} \beta_{n},
$$

where $\beta_{1}=\{(x, x) \mid x \in H\}$ is the diagonal relation on $H$. Suppose that $\beta^{*}$ is the transitive closure of $\beta$. Then $\beta^{*}$ is the smallest strongly regular relation on $H$ [3]. In this case $\beta^{*}$ is called the fundamental equivalence relation on $H$. If $H$ is a hypergroup, then $\beta^{*}=\beta$.

Theorem 3.7. If $\hat{G}$ is a single power cyclic polygroup, then $\hat{G}$ is simple.

Proof. Let $N$ be a normal subpolygroup of $\hat{G}$. According to Lemma 2.11, $N_{\hat{G}}$ is an equivalence relation on $\hat{G}$ such that $\chi N_{\hat{G}} \psi$ if and only if $\chi \bar{\psi} \cap N \neq \varnothing$.

By Lemma 2.12 and Theorem 2.13, the set of all equivalence classes is a polygroup with hyperoperation $\odot$ defined as follows:

$$
N_{\hat{G}}(\chi) \odot N_{\hat{G}}(\psi)=N \chi \odot N \psi=\{N \varphi \mid \varphi \in \chi \psi\} .
$$

Using Theorem 2.9, $N_{\hat{G}}$ is a regular equivalence relation on $\hat{G}$. Now we prove that $N_{\hat{G}}$ is a strongly regular equivalence relation on $\hat{G}$. According to Theorem 2.10, it is enough to show that $N \chi \odot N \psi=N \varphi$ for all $\varphi \in \chi \psi$. Let $\varphi_{1}, \varphi_{2} \in \chi \psi$. We should prove that $\varphi_{1} N_{\hat{G}} \varphi_{2}$. Indeed, there exists $\rho \in N$ such that $\rho \in \varphi_{1} \overline{\varphi_{2}}$. Since $\varphi_{1}, \varphi_{2} \in \chi \psi, \chi \in \varphi_{1} \bar{\psi}$ and $\chi \in \varphi_{2} \bar{\psi}$. Hence

$$
\bar{\chi} \in \overline{\varphi_{2}} \psi \Rightarrow \chi \bar{\chi} \in \varphi_{1} \overline{\varphi_{2}} \psi \bar{\psi} \Rightarrow \chi_{1} \in \varphi_{1} \overline{\varphi_{2}} \psi \bar{\psi} \text {. }
$$


Since $\psi \bar{\psi} \subseteq N$, there exists $\sigma \in N$ such that $\chi_{1} \in \varphi_{1} \overline{\varphi_{2}} \sigma$. Hence $\bar{\sigma} \in \varphi_{1} \overline{\varphi_{2}}$. Now we set $\rho=\bar{\sigma}$. So $N_{\hat{G}}$ is a strongly regular equivalence relation on $\hat{G}$. On the other hand, $\hat{G}$ is a single power cyclic polygroup. Thus, there exists $\chi \in \hat{G}$ and $t \in \mathbb{N}$ such that $\hat{G}=\chi^{t}$. For every $\psi \in \hat{G}, \psi \in \underbrace{\chi * \chi_{*} * \ldots * \chi}_{t \text { times }}$, so $\beta^{*}(\psi)=\hat{G}$. On the other hand, $\beta^{*}$ is the smallest strongly regular relation on $\hat{G}$. Thus $\hat{G}=\beta^{*}(\psi) \subseteq N \psi$. So $N=\hat{G}$. Therefore, $\hat{G}$ is a simple polygroup.

Now we bring two theorems from [12].

Theorem 3.8 ([12], Theorem 3.4). For $n \geq 3, \hat{S_{n}}$ is a single power cyclic polygroup with respect to generator $\chi(g)=\mid$ fix $(g) \mid-1$. In fact, $\left(\hat{S_{n}}\right)=\chi^{n-1}$; for $n \geq 3$.

Theorem 3.9 ([12], Theorem 3.5). For $n \geq 4, \hat{A_{n}}$ is a single power cyclic polygroup with respect to generator $\chi \downarrow_{A_{n}}$. In fact, $\hat{A_{n}}=\left(\chi \downarrow_{A_{n}}\right)^{n-2}$; for $n \geq 4$.

Theorem 3.10. Let $G=\underbrace{S_{n} \times S_{n} \times \ldots \times S_{n}}_{t \text { times }}$ where $t \in \mathbb{N}$ and $n \geq 3$. Then the character polygroup $\hat{G}$ is a simple and single power cyclic polygroup.

Proof. According to Theorem 2.3, we know that the irreducible characters of $G$ are $\chi_{i_{1}} \times \chi_{i_{2}} \times \ldots \times \chi_{i_{t}}$, where $\chi_{i_{j}} \in \hat{S}_{n}$ and

$$
\chi_{i_{1}} \times \chi_{i_{2}} \times \ldots \times \chi_{i_{t}}\left(g_{i_{1}}, g_{i_{2}}, \ldots, g_{i_{t}}\right)=\chi_{i_{1}}\left(g_{i_{1}}\right) \chi_{i_{2}}\left(g_{i_{2}}\right) \ldots \chi_{i_{t}}\left(g_{i_{t}}\right) .
$$

Using Theorem 3.8, $\hat{S}_{n}$ is a single power cyclic polygroup with respect to $\chi$ where $\chi(g)=|f i x(g)|-1 \forall g \in S_{n}$. So $\forall \chi_{i} \in \hat{S}_{n}, \chi_{i} \in \chi^{n-1}$. It follows that

$$
\chi_{i_{1}} \times \chi_{i_{2}} \times \ldots \times \chi_{i_{t}} \subseteq \underbrace{\chi^{n-1} \times \chi^{n-1} \times \ldots \times \chi^{n-1}}_{t \text { times }}
$$

and

$$
\underbrace{\chi^{n-1} \times \chi^{n-1} \times \ldots \times \chi^{n-1}}_{t \text { times }}=(\underbrace{\chi \times \chi \times \ldots \times \chi}_{t \text { times }})^{n-1} .
$$

We denote $(\underbrace{\chi \times \chi \times \ldots \times \chi}_{t \text { times }})^{n-1}$ with $\psi$ as an irreducible character of $G$.

Hence $\hat{G}=(\psi)^{n-1}$ is a single power cyclic polygroup. Now using Theorem $3.7, \hat{G}$ is a simple polygroup and the proof is complete.

Theorem 3.11. Let $G=\underbrace{A_{n} \times A_{n} \times \ldots \times A_{n}}_{t \text { times }}$ where $t \in \mathbb{N}$ and $n \geq 4$. Then the character polygroup $\hat{G}$ is a simple and single power cyclic polygroup.

Proof. Using Theorem 3.9, in the same manner as in Theorem 3.10, we can see that $\hat{G}=\left(\psi \downarrow_{G}\right)^{n-2}$ is a simple and single power cyclic polygroup. 


\section{CYCLIC CHARACTER POLYGROUPS}

In this section, we prove that if $G$ is a non-abelian simple group, then $\hat{G}$ is a single power cyclic polygroup. Also, we show that the polygroups $\hat{T}_{4 n}$ and $\hat{U}_{6 n}$ are cyclic with finite period.

Proposition 4.1. If $G$ is a non-abelian simple group, then $\hat{G}$ is a simple and single power cyclic polygroup.

Proof. By Theorem 2.8, we observe that $\hat{G}$ is a simple polygroup. On the other hand, all irreducible characters of $G$ are faithful and the order of $G$ is an even integer. Thus $G$ has a real class, and hence $G$ has at least one real irreducible character $\chi$. Then

$$
\chi=\bar{\chi} \Rightarrow 1=(\chi, \chi)=(\chi, \bar{\chi})=\left(\chi^{2}, \chi_{1}\right) \Rightarrow \chi_{1} \in \chi^{2} .
$$

So we have

$$
\chi_{1} \in \chi^{2} \subseteq \chi^{4} \subseteq \chi^{6} \subseteq \ldots
$$

Since $G$ is simple, $\chi^{2}$ is faithful. Now suppose that $\chi^{2}(g)$ takes on exactly $m$ different values for $g \in G$. Then by Theorem $2.2, \operatorname{Irr}(G)=\chi_{1} \cup \chi^{2} \cup\left(\chi^{2}\right)^{2} \cup \ldots \cup$ $\left(\chi^{2}\right)^{m-1}$. So $\hat{G}=\operatorname{Irr}(G)=\left(\chi^{2}\right)^{m-1}$ for some $m$. Therefore, $\hat{G}$ is single power cyclic.

Theorem 4.2. Let $G=T_{4 n}$. Then $\hat{G}$ is a cyclic polygroup with finite period respect to $\psi_{1}$. In fact, $\hat{G}=\psi_{1}^{n-1} \cup \psi_{1}^{n}$.

ProOF.

First, let $n$ be an even integer. Using Tables 3 and ??, we prove that for $1 \leq i \leq 4, \chi_{i}$ is a constituent of $\psi_{1}^{n}$. Since $n$ is an even integer, then $\psi_{1}{ }^{n}$ is a positive real character, and thus $\left(\psi_{1}^{n}, \chi_{j}\right)>0$ for $j=1,2$. Therefore, $\chi_{1}, \chi_{2} \in \psi_{1}^{n}$.

Now for $\chi_{3}$ we have:

$$
\begin{aligned}
& \left(\psi_{1}^{n}, \chi_{3}\right)=\frac{2^{n}+2^{n}}{4 n}+\frac{-\left(\epsilon+\epsilon^{-1}\right)^{n}+\left(\epsilon^{2}+\epsilon^{-2}\right)^{n}-\cdots-\left(\epsilon^{n-1}+\epsilon^{-(n-1)}\right)^{n}}{2 n} \\
& =\frac{2^{n}-\sum_{k=0}^{n} \epsilon^{-n+2 k}+\sum_{k=0}^{n} \epsilon^{-2 n+4 k}-\cdots-\sum_{k=0}^{n} \epsilon^{-n^{2}+n+2 n k-2 k}}{2 n}
\end{aligned}
$$

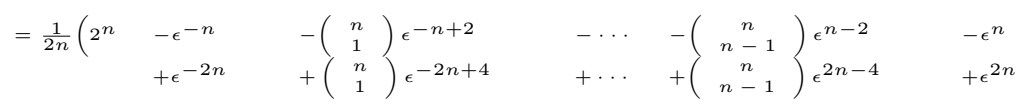

$$
\begin{aligned}
& \left.-\epsilon^{-n^{2}+n} \quad-\left(\begin{array}{c}
n \\
1
\end{array}\right) \epsilon^{-n^{2}+3 n-2} \quad-\cdots \quad-\left(\begin{array}{c}
n \\
n-1
\end{array}\right) \epsilon^{n^{2}-3 n+2} \quad-\epsilon^{n^{2}-n}\right)
\end{aligned}
$$

In the last equality we define numbers $A_{k}$ and $B_{n-k}$ for $0 \leq k<\frac{n}{2}$ as follow:

$$
\begin{aligned}
& A_{k}=-\epsilon^{-n+2 k}+\epsilon^{-2 n+4 k}-\ldots-\epsilon^{-n^{2}+2 n k+n-2 k}, \\
& B_{n-k}=-\epsilon^{n-2 k}+\epsilon^{2 n-4 k}-\ldots-\epsilon^{n^{2}-2 n k-n+2 k} .
\end{aligned}
$$

So we have:

$$
A_{\frac{n}{2}}=B_{\frac{n}{2}}=-1, A_{0}+B_{n}=2(n-1) .
$$


For $k=1$ we have:

$A_{1}+B_{n-1}=-\epsilon^{-n+2}-\epsilon^{n-2}+\epsilon^{-2 n+4}+\epsilon^{2 n-4}-\ldots-\epsilon^{(n-2)(n-1)}-\epsilon^{-(n-2)(n-1)}$.

On the other hand, using column orthogonality relations for $a^{n}, a^{n-2}$ we have:

$4-2\left(\epsilon^{-n+2}+\epsilon^{n-2}\right)+2\left(\epsilon^{-2 n+4}+\epsilon^{2 n-4}\right)-\ldots-2\left(\epsilon^{(n-2)(n-1)}+\epsilon^{-(n-2)(n-1)}\right)=0$.

So $A_{1}+B_{n-1}=-2$. We now apply this argument again, to obtain $A_{k}+B_{n-k}=-2$ for each $0 \leq k<\frac{n}{2}$ and $k \neq 0$. Hence

$$
\left(\psi_{1}^{n}, \chi_{3}\right)=\frac{1}{2 n}\left(2^{n}+2(n-1)-\left(\begin{array}{c}
n \\
\frac{n}{2}
\end{array}\right)-2 \sum_{k=1}^{\frac{n}{2}-1}\left(\begin{array}{c}
n \\
k
\end{array}\right)\right) .
$$

We can check that

$$
-2 \sum_{k=1}^{\frac{n}{2}-1}\left(\begin{array}{c}
n \\
k
\end{array}\right)=-2^{n}+\left(\begin{array}{c}
n \\
\frac{n}{2}
\end{array}\right)+2
$$

Thus

$$
\left(\psi_{1}^{n}, \chi_{3}\right)=1
$$

Therefore, $\chi_{3} \in \psi_{1}^{n}$, and similarly $\chi_{4} \in \psi_{1}^{n}$.

Now let $j$ be an even integer. Then we have

$$
\begin{aligned}
\left(\psi_{1}^{n}, \psi_{j}\right)= & \frac{2^{n+1}+2^{n+1}}{4 n}+ \\
& \frac{\left(\epsilon+\epsilon^{-1}\right)^{n}\left(\epsilon^{j}+\epsilon^{-j}\right)+\cdots+\left(\epsilon^{(n-1)}+\epsilon^{-(n-1)}\right)^{n}\left(\epsilon^{(n-1) j}+\epsilon^{-(n-1) j}\right)}{2 n} \\
= & \frac{2^{n+1}}{2 n}+\frac{\sum_{k=0}^{n} \epsilon^{-n+2 k+j}}{2 n}+\frac{\sum_{k=0}^{n} \epsilon^{-n+2 k-j}}{2 n}+\cdots+ \\
& \frac{\sum_{k=0}^{n} \epsilon^{-n^{2}+n(1+j)-j+2 k(n-1)}}{2 n}+\frac{\sum_{k=0}^{n} \epsilon^{-n^{2}+n(1-j)+j+2 k(n-1)}}{2 n}
\end{aligned}
$$

Last part of the above equality can be written as follows:

$$
\begin{aligned}
& \frac{1}{2 n}\left(2^{n+1}+\epsilon^{-n+j}+\left(\begin{array}{c}
n \\
1
\end{array}\right) \epsilon^{-n+2+j}+\cdots+\left(\begin{array}{c}
n \\
n-1 \\
1
\end{array}\right) \epsilon^{n-2+j}+\epsilon^{-2 n+4+2 j}+\epsilon^{n+j}\right. \\
& +\epsilon^{-2 n+2 j}+\left(\begin{array}{c}
n \\
1
\end{array}\right) \epsilon^{-2 n+4+2 j}+\cdots+\left(\begin{array}{c}
n-1 \\
n \\
n-1
\end{array}\right) \epsilon^{2 n-4+2 j}+\epsilon^{2 n+2 j}
\end{aligned}
$$

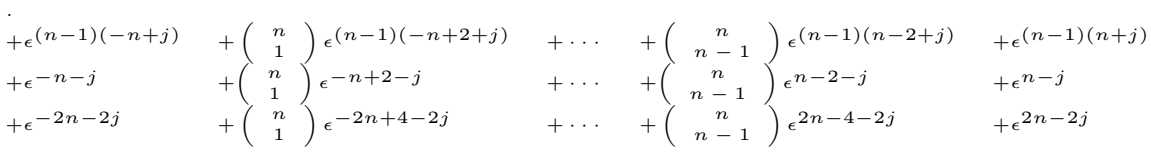

$$
\begin{aligned}
& \left.+\epsilon^{(n-1)(-n-j)}+\left(\begin{array}{c}
n \\
1
\end{array}\right) \epsilon^{(n-1)(-n+2-j)}+\cdots+\left(\begin{array}{c}
n \\
n-1
\end{array}\right) \epsilon^{(n-1)(n-2-j)}+\epsilon^{(n-1)(n-j)}\right) .
\end{aligned}
$$
as follow:

For all $0 \leq k \leq \frac{n}{2}$ and $k \neq \frac{n-j}{2}$ we define numbers $A_{k}, A_{k}^{\prime}, B_{n-k}$ and $B_{n-k}^{\prime}$

$$
\begin{aligned}
& A_{k}=\epsilon^{-n+2 k+j}+\epsilon^{-2 n+4 k+2 j}+\ldots+\epsilon^{-n^{2}+2 n k+n-2 k+n j-j}, \\
& A_{k}^{\prime}=\epsilon^{-n+2 k-j}+\epsilon^{-2 n+4 k-2 j}+\ldots+\epsilon^{-n^{2}+2 n k+n-2 k-n j+j}, \\
& B_{n-k}=\epsilon^{n-2 k+j}+\epsilon^{2 n-4 k+2 j}+\ldots+\epsilon^{n^{2}-2 n k-n+2 k+n j-j},
\end{aligned}
$$


$B_{n-k}^{\prime}=\epsilon^{n-2 k-j}+\epsilon^{2 n-4 k-2 j}+\ldots+\epsilon^{n^{2}-2 n k-n-2 k-n j+j}$.

Analysis similar to the case of even $n$, shows that for each $k$,

$$
A_{k}+B_{n-k}^{\prime}=-2\left(\begin{array}{c}
n \\
k
\end{array}\right), A_{k}^{\prime}+B_{n-k}=-2\left(\begin{array}{c}
n \\
k
\end{array}\right), k \neq \frac{n-j}{2} .
$$

And for $k=\frac{n-j}{2}$, we have that each component of $A_{k}$ and $B_{n-k}$ is equal to one.

Hence,

$$
\left(\psi_{1}^{n}, \psi_{j}\right)=\frac{1}{2 n}\left(2^{n+1}+2(n-1)\left(\begin{array}{c}
n \\
\frac{n-j}{2}
\end{array}\right)-2 \sum_{k=0}^{n}\left(\begin{array}{c}
n \\
k
\end{array}\right)+2\left(\begin{array}{c}
n \\
\frac{n-j}{2}
\end{array}\right)\right) .
$$

Also, we have

$$
-2 \sum_{k=0}^{n}\left(\begin{array}{c}
n \\
k
\end{array}\right)=-2(1+1)^{n}=-2\left(2^{n}\right)=-2^{n+1} .
$$

So

$$
\left(\psi_{1}^{n}, \psi_{j}\right)=\frac{1}{2 n} \cdot 2 n\left(\begin{array}{c}
n \\
\frac{n-j}{2}
\end{array}\right)=\left(\begin{array}{c}
n \\
\frac{n-j}{2}
\end{array}\right) .
$$

For odd integer $j$, similarly, we can check that $\psi_{j} \in \psi_{1}{ }^{n-1}$. Now let $n$ be an odd integer. Using Tables 3 and 5, similar to above, we can show that $\chi_{1}, \chi_{2}$ and $\psi_{j}$ for even integer $j$ are constituents of $\psi_{1}{ }^{n-1}$ and $\chi_{3}, \chi_{4}$ and $\psi_{j}$ for odd integer $j$ are constituents of $\psi_{1}^{n}$ and the proof is complete.

Theorem 4.3. Let $G=S_{3} \times C_{n}$ for odd integer $n$. Then $\hat{G}$ is a cyclic polygroup with finite period respect to $\psi_{3,1}$. In fact, $\hat{G}=\left(\psi_{3,1}\right)^{2} \cup\left(\psi_{3,1}\right)^{3} \cup \ldots \cup\left(\psi_{3,1}\right)^{n+1}$, where $\psi_{3,1}$ is the direct product of $\chi_{3}$ and $\varphi_{1}$.

Proof. We know that the character tables of $S_{3}$ and $C_{n}$ are as follow:

TABLE 7 . character table of $S_{3}$

\begin{tabular}{c|ccc} 
Class & 1 & $(12)$ & $(123)$ \\
\hline$\left|C_{S_{3}}\left(g_{i}\right)\right|$ & 6 & 2 & 3 \\
\hline$\chi_{1}$ & 1 & 1 & 1 \\
$\chi_{2}$ & 1 & -1 & 1 \\
$\chi_{3}$ & 2 & 0 & -1
\end{tabular}

TABLE 8. character table of $C_{n}$

\begin{tabular}{c|c} 
Class & $a^{r}(0 \leq r \leq n-1)$ \\
\hline$\left|C_{C_{n}}\left(g_{i}\right)\right|$ & $n$ \\
\hline$\varphi_{j}(1 \leq j \leq n)$ & $w^{r j}$
\end{tabular}


TABLE 9. character table of $G=S_{3} \times C_{n}$

\begin{tabular}{c|ccc} 
Class & $\left(1, a^{r}\right)$ & $\left((12), a^{r}\right)$ & $\left((123), a^{r}\right)$ \\
\hline$\left|C_{G}\left(g_{i}\right)\right|$ & $6 n$ & $2 n$ & $3 n$ \\
\hline$\psi_{1, j}=\chi_{1} \times \varphi_{j}$ & $w^{r j}$ & $w^{r j}$ & $w^{r j}$ \\
$\psi_{2, j}=\chi_{2} \times \varphi_{j}$ & $w^{r j}$ & $-w^{r j}$ & $w^{r j}$ \\
$\psi_{3, j}=\chi_{3} \times \varphi_{j}$ & $2 w^{r j}$ & 0 & $-w^{r j}$
\end{tabular}

Where $w$ is a primitive nth root of unity. So the character table of $G$ is as follows:

Now we prove that for $1 \leq t \leq 3$ and $2 \leq j \leq n, \psi_{t, j}$ and $\psi_{t, 1}$ are some constituents of $\left(\psi_{3,1}\right)^{j}$ and $\left(\psi_{3,1}\right)^{n+1}$, respectively.

First, let $t=1$ and $j$ be an even integer. Then

$$
\begin{aligned}
\left(\left(\psi_{3,1}\right)^{j}, \psi_{1, j}\right)= & \frac{2^{j}}{6 n}\left(1+w^{2 j}+w^{4 j}+\ldots+w^{2(n-1) j}\right)+ \\
& \frac{1}{3 n}\left(1+w^{2 j}+w^{4 j}+\ldots+w^{2(n-1) j}\right) .
\end{aligned}
$$

But $\left(\varphi_{j}, \varphi_{j}\right)=1$. So $1+w^{2 j}+w^{4 j}+\ldots+w^{2(n-1) j}=n$.

Then

$$
\left(\left(\psi_{3,1}\right)^{j}, \psi_{1, j}\right)=\frac{2^{j-1}+1}{3} .
$$

Similarly for odd integer $j$ we have

$$
\left(\left(\psi_{3,1}\right)^{j}, \psi_{1, j}\right)=\frac{2^{j-1}-1}{3} .
$$

Also,

$$
\begin{aligned}
\left(\left(\psi_{3,1}\right)^{n+1}, \psi_{1,1}\right)= & \frac{2^{n+1}}{6 n}\left(1+w^{n+2}+w^{2 n+4}+\ldots+w^{(n-1)(n+2)}\right)+ \\
& \frac{1}{3 n}\left(1+w^{n+2}+w^{2 n+4}+\ldots+w^{(n-1)(n+2)}\right) \\
= & \frac{2^{n+1} n}{6 n}+\frac{n}{3 n}=\frac{2^{n}+1}{3} .
\end{aligned}
$$

For other cases, the proof is the same as $t=1$.

Corollary 4.4. Let $n$ be an odd integer. Then $U_{6 n} \cong S_{3} \times C_{n}$, and thus $\hat{U}_{6 n}$ is a cyclic polygroup with finite period.

Proof. It is clear that the mapping $a \mapsto((12), w)$ and $b \mapsto((123), 1)$ is an isomorphism between $U_{6 n}$ and $S_{3} \times C_{n}$ and the proof is complete.

Theorem 4.5. Let $n$ be an even integer. Then $\hat{U}_{6 n}$ is a cyclic polygroup with finite period respect to $\psi_{1}$. In fact, $\hat{U}_{6 n}=\psi_{1}^{2} \cup \psi_{1}^{3} \cup \ldots \cup \psi_{1}^{n+1}$. 
Proof. According to Table 2.6, we see that $U_{6 n}$ has $n$ irreducible characters $\psi_{k}$ of degree 2. It is clear that $\psi_{0} \in \psi_{1}^{n}$ and $\psi_{1} \in \psi_{1}{ }^{n+1}$. We show that for $1<k \leq n-1, \psi_{k} \in \psi_{1}{ }^{k}$. Let $k$ be an even integer. Then

$$
\begin{aligned}
\left(\psi_{k}, \psi_{1}{ }^{k}\right)= & \frac{2^{k+1}}{6 n}\left(1+w^{2 k+2 k}+w^{2(2 k+2 k)}+\ldots+w^{(n-1)(2 k+2 k)}\right)- \\
& \frac{1}{3 n}\left(1+w^{2 k+2 k}+w^{2(2 k+2 k)}+\ldots+w^{(n-1)(2 k+2 k)}\right) .
\end{aligned}
$$

But $\left(\psi_{k}, \psi_{k}\right)=1$. Hence

$$
1+w^{2 k+2 k}+w^{2(2 k+2 k)}+\ldots+w^{(n-1)(2 k+2 k)}=n .
$$

Then

$$
\left(\psi_{k}, \psi_{1}^{k}\right)=\frac{2^{k+1} n}{6 n}-\frac{n}{3 n}=\frac{2^{k}-1}{3} .
$$

When $k$ is an odd integer, similar to above, we can prove that

$$
\left(\psi_{k}, \psi_{1}{ }^{k}\right)=\frac{2^{k}+1}{3} .
$$

Also, $U_{6 n}$ has $2 n$ linear irreducible characters $\chi_{j}$. It is easy to show that $\left(\chi_{0}, \psi_{1}{ }^{n}\right)=\frac{2^{n-1}+1}{3}$ and $\left(\chi_{1}, \psi_{1}{ }^{n+1}\right)=\frac{2^{n+1}-2}{3}$. So $\chi_{0} \in{\psi_{1}}^{n}$ and $\chi_{1} \in \psi_{1}{ }^{n+1}$. Now We show that for $1<j \leq 2 n-1, \chi_{j} \in \psi_{1}{ }^{s}$ where $s$ is the smallest positive integer that $n \mid j+s$. First, let $j$ be an even integer. Then

$$
\begin{aligned}
\left(\chi_{j}, \psi_{1}^{s}\right)= & \frac{2^{s}}{6 n}\left(1+w^{2 j+2 s}+w^{2(2 j+2 s)}+\ldots+w^{(n-1)(2 j+2 s)}\right)+ \\
& \frac{1}{3 n}\left(1+w^{2 j+2 s}+w^{2(2 j+2 s)}+\ldots+w^{(n-1)(2 j+2 s)}\right) \\
= & \frac{2^{s} n}{6 n}+\frac{n}{3 n}=\frac{2^{s-1}+1}{3} .
\end{aligned}
$$

When $j$ is an odd integer, similarly, we can check that

$$
\left(\chi_{j}, \psi_{1}^{s}\right)=\frac{2^{s-1}-1}{3} .
$$

The proof is complete.

\section{CONCLUSION}

In this paper, we investigate some special polygroups in terms of simplicity and circularity and study the relation between single power cyclic polygroups and simple polygroups. These results raise the following problems:

"Under what conditions a simple polygroup is going to be a single power cyclic polygroup? Is there a special class of finite groups for which the character polygroups are simple?" 
Acknowledgement. The authors express their gratitude to the referees for very useful comments and suggestions. This work was partially supported by the Center of Excellence of Algebraic Structures and its Applications of Tarbiat Modares University (CEAS).

\section{REFERENCES}

[1] Brauer, R., On pseudo groups, J. Math. Soc. Japan, 20 (1968), 13-22.

[2] Comer, S.D., Hyperstructures associated with character algebra and color schemes, New Frontiers in Hyperstructures, Hadronic Press, (1996) 49-66.

[3] Corsini, P., Prolegomena of Hypergroup Theory, Aviani Editore, Tricesimo, analy 1993.

[4] Davvaz, B., Polygroup Theory and Related Systems, World Scientific Publishing co. Pte. Ltd, 2013.

[5] James, G., Liebeck, M. W., and Liebeck, M., Representations and characters of groups, Cambridge University Press, 2001.

[6] Konguetsof, L., Vougiouklis, T., Kessoglides, M. and Spartalis, S., On cyclic hypergroups with period, Acta Univ. Carolinae-Math. Physica., 28 (1987), no 1, 3-7.

[7] Koskas, M., Groupoides, demi-hypergroups et hypergroupes, J. Math. Pures Appl., 49 (1970), 155-192.

[8] Leoreanu, V., About the simplifiable cyclic semihypergroups, Italian J. Pure Appl. Math., 7 (2000), 69-76.

[9] Marty, F., "Sur une generalization de la notion de group" in Proc. 8th Congress Mathematiciens Scandenaves, Stockholm, Sweden (1934), 45-49.

[10] Mittas, J., Hypergroupes canoniques hypervalues (French), C. R. Acad. Sci. Paris Ser, A-B, 271 (1970), A4- A-7.

[11] Roth, R.L., Character and conjugacy class hypergroups of a finite group, Ann. Math. Pura Appl., 105 (1975), 295-311.

[12] Sekhavatizadeh, S., Zahedi, M.M. and Iranmanesh, A., Cyclic hypergroups which are induced by the character of some finite groups, Italian J. Pure Appl. Math. 33 (2014), 123-132.

[13] Vougiouklis, T., Cyclicity in a special class of hypergroups, Acta Univ. Carolinae-Math. et Physica., 22 (1981), 3-6.

[14] Vougiouklis, T., Hyperstructures and therir Representations, Hadronic Press, Palm Harbor, FL, 1994.

[15] Wall, H.S., Hypergroups, Amer. J. Math., 59 (1937), 77-98. 